8. Pawel Wiech, Zdzislawa Chmiel, Dariusz Bazalinski et al (2021). Body Composition and Selected Nutritional Indicators in Healthy Adults-A Cross-Sectional Study. Glob Adv Health Med, 2021 Jun 3; Vol 10: 1-13.
9. Susan Keino, Bart van den Borne and Guy Plasqui (2014). Body composition, water turnover and physical activity among women in Narok County, Kenya. PMC Public Health, 2014 Nov 24; $14: 121$

\title{
NHẬN XÉT GIÁ TRI CỦA PET/CT TRONG ĐÁNH GIÁ DI CĂN PHỔI Ở BÊ̂NH NHÂN UNG THƯ ĐẠI TRỰC TRÀNG
}

\section{TÓM TẮT}

Mục tiêu: Nhân xét giá trị của PET/CT trong đánh giá di căn phổi ở bệnh nhân ung thư đại trực tràng đã điều trị. Đối tượng và phương pháp nghiên cứu: 39 bệnh nhân (18 bệnh nhân ung thư đại tràng và 21 bệnh nhân ung thư trực tràng) đã điều trị triệt căn có nốt tổn thương tại phổi được chụp CT cản quang và PET/CT (với ${ }^{18} \mathrm{~F}-\mathrm{FDEG}$ ) đánh giá tại bệnh viện Ung bướu Hà Nội từ tháng 10/2019 đến tháng 5/2020. Nghiên cứu mô tả cắt ngang, hồi cứu kết hợp tiến cứu. Kết quả: Tuổi trung bình của bệnh nhân trong nhóm nghiên cứu là $60,1 \pm 13,3$. 38, $5 \%$ số bệnh nhân có nồng độ CEA bình thường. PET/CT phát hiện số tổn thương dương tính nhiêuu hơn so với CT (48 so với 39). Đa số các trường hợp di căn phổi trong nhóm nghiên cứu có di căn nhiều nốt với kích thước nhỏ, giá trị SUVmax không cao. Kích thước trung bình các tổn thương tại phổi là $1,0 \pm 1,5 \mathrm{~cm}$. Giá trị SUVmax trung bình chung của cả nhóm nghiên cứu là 3,19 $\pm 2,23$. PET/CT có độ nhạy $(95,7 \%)$ và độ đăc hiệu $(62,5 \%)$ trong đánh giá các nốt tại phổi. Kết luận: PET/CT phát hiện nhiều tổn thương dương tính tại phổi hơn $\mathrm{CT}$ với đô nhay và độ đăc hiêu của chẩn đoán là $95,7 \%$ và $62,5 \%$. Tuy nhiệ̂n với giá trị dự báo dương tính cao, chi phí thấp và dễ thực hiện, $\mathrm{CT}$ vẫn hữu ích trong thực hành lâm sàng.

\section{SUMMARY}

TO REVIEW THE ROLE OF PET/CT IN THE EVALUATION OF LUNG METASTASES IN

THE COLORECTAL CANCER PATIENTS

Objective: To review the role of PET/CT in the evaluation of lung metastases in the colorectal cancer patients who had had undergone curative treatment. Material and methods: 39 patients (18 colon cancer and 21 rectal cancer) who had undergone curative treatment with pulmonary nodules were evaluated by contrast CT and PET/CT (with ${ }^{18} \mathrm{~F}-\mathrm{FDG}$ ) at Hanoi Oncology Hospital from October 2019 to May 2020. A

\footnotetext{
${ }^{1}$ Bệnh viện Ung bướu Hà Nội

2Trung tầm CĐHA và YHHN - bênh viên $K$

${ }^{3}$ Trường Đại họ Y Hà Nội, Trung tâm YHHN và UB Bênh viên Bach Mai

Chịu trách nhiệm chính: Nguyễn Văn Khải

Email: nguyen vankhai.ubhn@gmail.com

Ngày nhân bài: 5.8 .2021

Ngày phản biên khoa hoc: 4.10 .2021

Ngày duyệt bài: 14.10.2021
}

retrospective and prospective descriptive study. Results: The average age of patients in the study was $60,1 \pm 13,3$. 38,5\% of patients had normal CEA levels. PET/CT detected more positive lesions than CT (48 vs 39). Most of the cases of lung metastasis in the study group had metastases with many small nodules, SUVmax values were not high. The average size of pulmonary lesions was $1,0 \pm 1,5 \mathrm{~cm}$. The overall value of SUVmax of the group was 3,19 $\pm 2,23$. PET/CT has sensitivity $(95,7 \%)$ and specificity $(62,5 \%)$ in evaluating pulmonary nodules. Conclusion: PET/CT detected more positive pulmonary lesions than CT with diagnostic sensitivity and specificity of $95,7 \%$ and $62,5 \%$. However, with high positive predictive value, low cost and ease of implementation, CT remains useful in clinical practice.

Keywords: PET/CT, colorectal cancer, lung metastasis

\section{I. ĐẶT VẤN ĐỀ}

Ung thư đại trực tràng là một trong những loại ung thư có tỉ lệ mắc hàng đầu ở Việt Nam. Ngoài việc chẩn đoán, điều trị và đánh giá đáp ứng sau điều trị thì việc theo dõi phát hiện bệnh tái phát, di căn cũng có một vai trò quan trọng. Việc phát hiên sớm hay đánh giá đúng tổn thương có thể làm thay đổi chiến thuật, đưa ra các quyết định điêu trị phù hợp. UTĐTT di căn cơ quan xa phổ biến nhất đến phổi và tiếp sau là gan, tỉ lệ di căn phổi lên đến 15\% [1]. Với hình ảnh điển hình di căn phổi trên $C T$, việc chẩn đoán không quá khó khăn. Tuy nhiên, bệnh nhân UTĐTT được xác định có nốt ở đơn độc ở phổi trong trường hợp không có di căn khác là một tình trạng gây ra rất nhiều khó khăn cho bác sĩ lâm sàng trong việc phải đưa ra quyết định lựa chọn điều trị tiếp theo. Nhiều nghiên cứu đã được thực hiện so sánh khả năng của các kỹ thuât hình ảnh khác nhau để xác định đây đủ các đăc điểm của nốt đơn độc tại phổi là lành tính hay ác tính. Hiện nay hai kỹ thuật chính hiện được sử dụng để đánh giá là $\mathrm{CT}$ cán quang và $\mathrm{PET} / \mathrm{CT}$, vì vậy chúng tôi tiến hành nghiên cứu này nhằm mục tiêu nhận xét giá trị của $\mathrm{PET} / \mathrm{CT}$ trong đánh giá di căn phổi ở bệnh nhân ung thư đại trực tràng đã điều trị và so sánh với CT ngực có tiêm thuốc cản quang. 
II. ĐỐI TƯợNG VÀ PHƯƠNG PHÁP NGHIÊN CỨU

Đối tượng nghiên cứu: 39 bệnh nhân UTĐTT được chẩn đoán xác định bằng mô bệnh học đã điều trị có nốt tổn thương ở phổi được phát hiện trên hình ảnh Xquang hoặc CT ngực được chụp PET/CT với ${ }^{18}$ F-FDG để đánh giá. Các trường hợp phát hiện tổn thương phổi trên Xquang được chụp thêm CT cản quang.

- Thiết kế nghiên cứu: Nghiên cứu mô tả cắt ngang, hồi cứu kết hợp tiến cứu.

- Thời gian và địa điểm nghiên cứu: Từ tháng 10/2019 đến tháng $5 / 2020$ tại Khoa $Y$ học hạt nhân - Bệnh viện Ung bướu Hà Nội.

- Xử lý và phân tích số liệu: Thống kê, xử lý số liệu bằng phần mềm SPSS 25.

- Tiêu chuẩn hình ảnh PET/CT áp dụng trong nghiên cứu

+ Tổn thương dương tính: nốt mờ tại phổi tăng hấp thu FDG (SUVmax $\geq 2,5$ ) hoặc hình ảnh di căn phổi điển hình trên $\mathrm{CT}$ : nhiều nốt, các nốt di căn có kích thước khác nhau và được xác định rõ ràng, bờ nhẵn hoặc không đều, thường ưu thế ở thùy dưới, dưới màng phổi, phân bố ngẫu nhiên, có thể tăng hoặc không tăng hấp thu FDG.

+Tổn thương dương tính thật: tổn thương tăng hấp thu FDG được khẳng định bằng kêt quả mô bệnh học, đáp ứng với điều trị đặc hiệu và/hoặc theo dõi lâm sàng, hình ảnh trong 1 năm khẳng định di căn.

+ Tổn thương dương tính giả: tổn thương tăng hấp thu FDG nhưng kết quả giải phẫu bệnh không phải là ung thư và/hoặc thay đổi hay mất đi trong quá trình theo dõi, điều trị với chẩn đoán khác.
+ Tổn thương âm tính: tổn thương được phát hiện nhưng không tăng hấp thu FDG (SUVVmax: $<2,5$ ).

+ Tổn thương âm tính thật: tổn thương không tăng hấp thu FDG được khẳng định âm tính bằng kết quả mô bệnh học, đáp ứng với điều trị đặc hiệu và/hoặc theo dỗi lâm sàng, hình ảnh trong 1 năm khẳng định lành tính.

+ Tổn thương âm tính giả: không tăng chuyển hóa FDG nhưng được khẳng định dương tính bằng kết quả giải phẫu bệnh và/hoặc theo dõi lâm sàng, hình ảnh khẳng định ác tính.

+ Với các trường hợp nhiều nốt tổn thương (> 3 nốt), lấy tổn thương lớn nhất để đo kích thước và giá trị SUVmax.

\section{KẾT QUẢ NGHIÊN CỨU}

Phát hiện tổn thương trên PET/CT

Bảng 3.1. So sánh sưr phát hiện tổn thương tại phổi cúa CT với PET/CT

\begin{tabular}{|c|c|c|}
\hline Đặc điểm & $\begin{array}{c}\text { Tốn thương } \\
\text { trên CT } \\
(\mathrm{n}=55)\end{array}$ & $\begin{array}{c}\text { Tốn thương } \\
\text { trên PET/CT } \\
(\mathrm{n}=55)\end{array}$ \\
\hline $\begin{array}{c}\text { Số BN phát hiện } \\
\text { tổn thương }\end{array}$ & 39 & 39 \\
\hline $\begin{array}{c}\text { Số tốn thương } \\
\text { dương tính }\end{array}$ & 39 & 48 \\
\hline $\begin{array}{c}\text { Số tốn thương } \\
\text { âm tính }\end{array}$ & 16 & 7 \\
\hline $\begin{array}{c}\text { Kích thước lớn } \\
\text { nhất }(\mathrm{cm})\end{array}$ & 2,3 & 2,6 \\
\hline $\begin{array}{c}\text { Kích thước nhỏ } \\
\text { nhất }(\mathrm{cm})\end{array}$ & 0,5 & 0,5 \\
\hline
\end{tabular}

Nhận xét: PET/CT phát hiện tốn thương dương tính nhiêu hơn so với CT (48 so với 39).

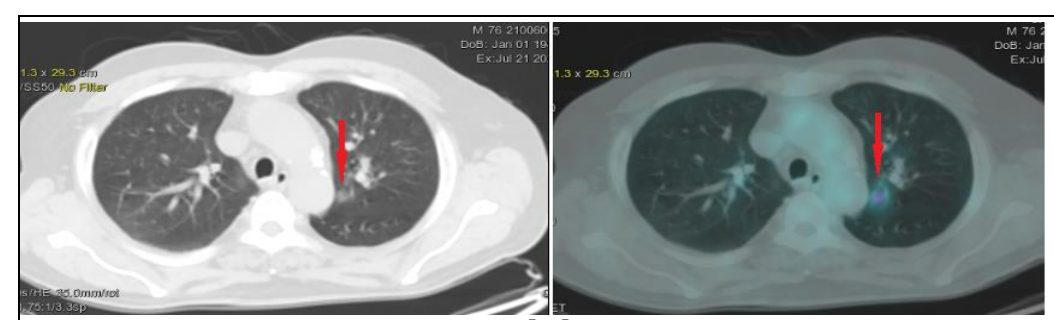

(A)

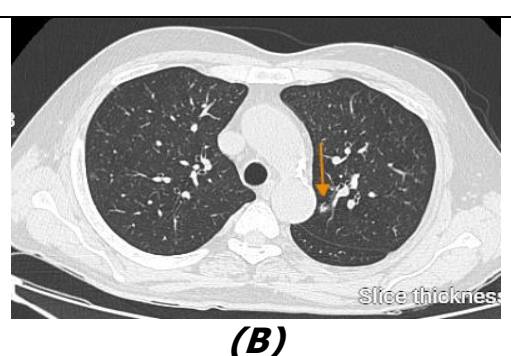

(B)

Hình 3.1. Tốn thương dương tính trên PET/CT (A). Trên hình ảnh CT phát hiện tốn thương tương ứng nhưng được đánh giá là âm tính (B).

- Tỉ lệ phát hiện tổn thương theo nồng độ CEA

Bảng 3.2. Ti lệ phát hiện tốn thương tại phổi theo nồng độ CEA

\begin{tabular}{|c|c|c|c|c|c|c|}
\hline \multicolumn{2}{|c|}{ Nồng độ CEA (ng/ml) } & $\begin{array}{c}<\mathbf{5} \\
(\mathbf{n = 1 5})\end{array}$ & $\begin{array}{c}\mathbf{5}-\mathbf{1 0} \\
(\mathbf{n}=\mathbf{1 3})\end{array}$ & $\begin{array}{c}\mathbf{1 0}-\mathbf{1 5} \\
\mathbf{( n = 2 )}\end{array}$ & $\begin{array}{c}>\mathbf{1 5} \\
(\mathbf{n}=\mathbf{9})\end{array}$ & $\begin{array}{c}\text { Tống } \\
(\mathbf{n = 3 9})\end{array}$ \\
\hline \multirow{2}{*}{ Dương tính } & Số BN & 12 & 12 & 2 & 8 & $\mathbf{3 4}$ \\
\cline { 2 - 7 } & Số tốn thương & 21 & 18 & 3 & 6 & $\mathbf{4 8}$ \\
\hline \multirow{2}{*}{ Âm tính } & Số BN & 3 & 1 & 0 & 1 & $\mathbf{5}$ \\
\cline { 2 - 7 } & Số tốn thương & 5 & 1 & 0 & 1 & $\mathbf{7}$ \\
\hline
\end{tabular}


Nhận xét: Số lượng tổn thương và số bệnh nhân phát hiện tổn thương âm tính và dương tính khác nhau ở các nhóm. Sự khác biệt không có ý nghĩa thống kề $(p>0,05)$.

- Giá trị SUVmax

Bảng 3.3. Giá trị SUVmax theo nồng độ CEA

\begin{tabular}{|c|c|c|c|c|c|}
\hline $\begin{array}{c}\text { Nồng độ CEA } \\
(\mathrm{ng} / \mathrm{m} l)\end{array}$ & $\begin{array}{c}<\mathbf{5} \\
(\mathrm{n}=15)\end{array}$ & $\begin{array}{c}\mathbf{5}-\mathbf{1 0} \\
(\mathrm{n}=13)\end{array}$ & $\begin{array}{c}\mathbf{1 0}-\mathbf{1 5} \\
(\mathrm{n}=2)\end{array}$ & $\begin{array}{c}>\mathbf{1 5} \\
(\mathrm{n}=9)\end{array}$ & $\begin{array}{c}\text { Tống } \\
(\mathrm{n}=39)\end{array}$ \\
\hline Số lượng tốn thương & 21 & 18 & 3 & 6 & 48 \\
\hline SUVmax trung bình & $3,14 \pm 1,71$ & $2,87 \pm 2,14$ & $4,23 \pm 3,91$ & $3,77 \pm 4,38$ & $3,19 \pm 2,23$ \\
\hline
\end{tabular}

Nhận xét: Giá trị SUVmax trung bình của tổn thương ở các nhóm có nông độ CEA khác nhau là khác nhau, tuy nhiên sự khác biệt không có ý nghĩa thống kê $(p>0,05)$.

Bảng 3.4. Giá trị SuVmax theo kích thước tổn thương

\begin{tabular}{|c|c|c|c|}
\hline Kích thước tốn thương $(\mathrm{cm})$ & $\mathbf{1}(\mathrm{n}=27)$ & $\mathbf{> 1}$ và $\leq \mathbf{2}(\mathrm{n}=13)$ & $\mathbf{>} \mathbf{2}$ và $\leq \mathbf{3}(\mathrm{n}=8)$ \\
\hline Kích thước trung bình & $0,6 \pm 0,1$ & $1,4 \pm 0,3$ & $2,2 \pm 0,2$ \\
\hline SUVmax trung bình & $1,72 \pm 1,58$ & $5,15 \pm 3,89$ & $4,94 \pm 0,83$ \\
\hline Kích thước trung bình chung & \multicolumn{3}{|c|}{$1,0 \pm 1,5$} \\
\hline SUVmax trung bình chung & \multicolumn{3}{|c|}{$3,19 \pm 3,23$} \\
\hline
\end{tabular}

Nhận xét: Đa số các trường hợp di căn ung thư phối trong nhóm nghiên cứu có di căn nhiều nốt kích thước nhỏ $(<1 \mathrm{~cm})$, giá trị SUVmax thấp. Kích thước trung bình các tổn thương tại phổi là $1,0 \pm$ $1,5 \mathrm{~cm}$. Giá trị SUVmax trung bình chung của cả nhóm nghiên cứu là $3,19 \pm 2,23$. Có sự khác biệt về giá trị SUVmax theo kích thước các nốt tổn thương tại phổi $(p=0,005$.



Hình 3.2. Hình ảnh di căn phổi trên PET/CT

(Di căn phổi với nhiều nốt nhỏ rải rác khắp phế trường, giá trị SUVmax của tổn thương không cao)

- Giá trị chẩn đoán

Bảng 3.5. Giá trị chẩn đoán của PET/CT

\begin{tabular}{|c|c|c|}
\hline Giá trị & $\begin{array}{c}\text { Tốn thương } \\
\text { trên CT } \\
(\mathrm{n}=55)\end{array}$ & $\begin{array}{c}\text { Tốn thương } \\
\text { trên PET/CT } \\
(\mathrm{n}=55)\end{array}$ \\
\hline Dương tính thật $(\mathrm{n})$ & 38 & 45 \\
\hline Dương tính giả $(\mathrm{n})$ & 1 & 3 \\
\hline Âm tính thật $(\mathrm{n})$ & 7 & 5 \\
\hline Âm tính giả $(\mathrm{n})$ & 9 & 2 \\
\hline Độ nhạy $(\%)$ & 80,9 & 95,7 \\
\hline Độ đặc hiệu (\%) & 87,5 & 62,5 \\
\hline $\begin{array}{c}\text { Dự báo dương tính } \\
(\%)\end{array}$ & 97,4 & 93,8 \\
\hline Dự báo âm tính(\%) & 43,8 & 71,4 \\
\hline
\end{tabular}

Nhận xét: 39 bệnh nhân với 55 tổn thương tại phổi được đối chiếu với kết quả mô bệnh học hoặc theo dõi lâm sàng, hình ảnh theo dṍi trong vòng 1 năm. Kết quả độ nhạy và độ đặc hiệu trong chẩn đoán tổn thương tại phổi của PET/CT là $95,7 \%$ và $62,5 \%$ tương ứng, ở $\mathrm{CT}$ là $80,9 \%$ và $87,5 \%$ tương ứng.

\section{BÀN LUẬN}

Kết quả nghiên cứu cho thây $\mathrm{PET} / \mathrm{CT}$ phát hiện số lượng tổn thương dương tính nhiều hơn so với CT cản quang. Đa số di căn phổi của UTĐTT là nhiêu nốt tổn thương nhỏ, có thể gặp ở một hay hai bên phổi, các trường hợp di căn nốt đớn độc ít gặp hơn. 7/32 trường hợp di căn các nốt nhỏ lan tỏa khắp phế trường. Kích thước các tổn thương cũng rất đa dạng. Trung bình ở các bệnh nhân là $1,0 \pm 1,5 \mathrm{~cm}$. Với các trường hợp di căn phổi điển hình trên hình ảnh $\mathrm{CT}$ thì không khó khăn trong chẩn đoán. Thông thường là di căn nhiều nốt, các nốt di căn có kích thước khác nhau và được xác định rõ ràng, bờ nhẵn hoặc không đều. Chúng chiếm ưu thế ở thùy dưới, dưới màng phổi. Trên CT độ phân giải cao, các nốt này được phân bố một cách ngẫu nhiên, không giống như các bệnh khác có sự phân bố theo quy luật hoặc phẩn bố ở trung tâm tiểu thùy. A.J. Quyn (2012) nghiên cứu di căn phổi trên 908 bệnh nhân UTĐTT nhận thấy một nửa số bệnh nhân có trên 4 nốt mờ tại phổi trở lên gợi ý tình trạng khả năng là di căn phổi [1].

Nhiều nghiên cứu đã được thực hiện so sánh khả năng của các kỹ thuật hình ảnh khác nhau để xác định đầy đủ các đặc điểm của nốt đơn độc tại phổi là lành tính hay ác tính. Nốt phổi trên CT tăng $>15 \mathrm{H}$ từ mức không cản quang đến mức ngấm thuốc cao nhất được coi là có khả năng ác tính, trong khi những tổn thương tăng < $15 \mathrm{H}$ được coi là lành tính. Một phân tích đa trung tâm cho thấy, CT bằng các tiêu chí này có độ nhạy là $98 \%$ và độ đặc hiệu là $58 \%$ [2]. Trong 
nghiên cứu của chúng tôi, độ nhạy và độ đặc hiệu của CT tương ứng là $80,9 \%$ và $87,5 \%$.

Nốt phổi trên hình ảnh PET/CT khi tăng hấp thu lớn hơn mức của bể máu trung thất có khả năng là ác tính. Một giá trị SUV lớn hơn 2,5 xác định là ác tính với độ nhạy và độ đặc hiệu tương đối cao [3]. Trong một nghiên cứu của Tang (2019) trên 166 nốt tổn thương, các tổn thương với giá trị SUVmax $<1,25$ là lành tính [4]. Với ngưỡng giá trị SUVmax $=2,5$, các nghiên cứu gần đây đã cho thấy độ nhạy 92-96\% và độ đặc hiệu là 77 -90\% khi sử dụng 18F-FDG PET [3]. Nghiên cứu của Christensen (2006) đánh giá các nốt mờ phổi có kích thước $>0,7 \mathrm{~cm}$ với 42 nốt được đánh giá, PET dương tính ở 24/25 nốt ác tính và ở $4 / 17$ nốt lành tính. Độ nhạy và độ đặc hiệu lần lượt là $96 \%$ và $76 \%$, so với CT với độ nhạy và độ đặc hiệu tương ứng là $100 \%$ và $29 \%$ [5]. Trong nghiên cứu của chúng tôi, giá trị SUVmax giới hạn cũng được sử dụng ở ngưỡng 2,5 với các tổn thương không điển hình di căn trên hình ảnh CT. Giá trị SUVmax trung bình ở nhóm nghiên cứu là $3,19 \pm 2,23$. Có sự khác biệt về giá trị SUVmax theo kích thước tổn thương ( $p$ $<0,005)$. Độ nhạy và độ đặc hiệu của chẩn đoán tương ứng là $95,7 \%$ và $62,5 \%$. Kết quả này tương tự 1 số nghiên cứu khác [4], [5].

Có nhiều nghiên cứu về ngưỡng giá trị SUVmax trong đánh giá các nốt tổn thương tại phổi (bảng 4.1).

Bảng 4.1. Giá trị chẩn đoán của PET/CT của các nghiên cứu

\begin{tabular}{|c|c|c|c|}
\hline Tác giả & Chúng tôi & $\begin{array}{c}\text { Degirmenci } \\
\text { (2008) [6] }\end{array}$ & $\begin{array}{c}\text { V.G López } \\
\text { (2015) [7] }\end{array}$ \\
\hline $\mathrm{N}$ & 55 & 49 & 55 \\
\hline $\begin{array}{c}\text { SUVmax } \\
\text { cut-off }\end{array}$ & 2,5 & 2,4 & 1,95 \\
\hline $\begin{array}{c}\text { Độ nhạy } \\
(\%)\end{array}$ & 95,7 & 62,0 & 80,0 \\
\hline $\begin{array}{c}\text { Độ đặc } \\
\text { hiệu (\%) }\end{array}$ & 62,5 & 80,0 & 53,5 \\
\hline
\end{tabular}

Mặc dù đưa ra các ngưỡng SUVmax khác nhau, nhưng các tác giả đều có một điểm chung là ngưỡng giá trị SUVmax nên được lựa chọn theo kích thước của tổn thương. Sang Mi Lee (2008) nghiên cứu đánh giá 55 nốt tổn thương di căn phổi được phẫu thuật khẳng định chẩn đoán trên 34 bệnh nhân UTĐTT nhận thấy độ chính xác chẩn đoán và giá trị giới hạn tốt nhất của SUVmax thay đổi theo kích thước của nốt. Giá trị SUVmax giới hạn tốt nhất là theo kích thước tổn thương, giá trị SUVmax giới hạn với các kích thước tổn thương $0,6-0,8 \mathrm{~cm}, 1,2-1,4 \mathrm{~cm}$ và $1,8-2 \mathrm{~cm}$ tương ứng là 1,$4 ; 2,3$ và 3,6 [8].
Bamba Y. (2011), đánh giá trên 256 bệnh nhân UTĐTT bằng $P E T / C T$, di căn phổi và nghi ngờ di căn phổi được phát hiện ở 37 bệnh nhân. Sự hấp thu FDG bị ảnh hưởng bởi kích thước của các nốt di căn; hấp thu dương tính và âm tính ở di căn phổi với kích thước trung bình lần lượt là $1,49 \mathrm{~cm}$ và $0,575 \mathrm{~cm}$. Giới hạn hấp thu $\mathrm{FDG}$ dương tính đạt được trong di căn phổi là khoảng $0,9 \mathrm{~cm}$. Di căn phổi của ung thư đại trực tràng có thể được chẩn đoán chính xác bằng $\mathrm{PET} / \mathrm{CT}$, đặc biệt khi các nốt $>0,9 \mathrm{~cm}$. Độ nhạy và độ đặc hiệu của PET/CT đối với di cắn phổi lần lượt là $57,1 \%$ và $99,1 \%$, giá trị dự báo dương tính và âm tính lần lượt là $90,1 \%$ và $93,6 \%$ [9]. Một nghiên cứu khác sử dụng tỉ số $\mathrm{T} / \mathrm{M}$ ( $\mathrm{T}$ : giá trị SUVmax của nốt mờ tại phổi, M: giá trị SUVmean của bể máu trung thất) trong đánh giá các nốt tại phổi cho thấy, khi lấy ngưỡng tỉ số $T / M=$ 2,13 , các tổn thương có tỉ số $\mathrm{T} / \mathrm{M}>2,13$ được coi là dương tính, độ nhạy và độ đặc hiệu của chẩn đoán là $56,9 \%$ và $62,1 \%$.

Dựa trên các kết quả trên, chúng tôi nhận thây, SUV là công cụ dự đoán tốt trong việc xác định di căn phổi do UTĐTT, nhưng giá trị SuVmax giới hạn phải được điều chỉnh theo kích thước của tổn thương để cho khả năng chẩn đoán tốt nhất. $\mathrm{PET}$ thích hợp hơn $\mathrm{CT}$ trong đánh giá các nốt phổi không xác định. Tuy nhiên, CT vẫn hữu ích do giá trị dự báo dương tính cao, tiện lợi và chi phí thấp hơn.

\section{KẾT LUÂN}

Qua nghiên cứu 39 bênh nhân ung thư đai trực tràng đã điều trị có tổn thương tại phổi được chụp CT ngực có tiêm thuốc cản quang và $\mathrm{PET} / \mathrm{CT}$ để đánh giá, chúng tôi rút ra một số kết luân sau:

Số lượng tổn thương dương tính tại phổi trên PET/CT cao hơn CT trong đánh giá các nốt tại phổi ở nhóm bệnh nhân nghiên cứu.

- PET/CT có đổ nhạy $(95,7 \%)$ trong đánh giá các nốt tại phổi cao hơn $\mathrm{CT}$ nhưng độ đặc hiệu thấp hơn (62,5\%). Với giá tri dự báo dương tính cao $(97,4 \%)$, chi phí thấp, dễ thực hiện, CT vẫn hữu ích trong thực hành lâm sàng.

\section{TÀI LIÊU THAM KHẢO}

1. A.J Quyn, A Matthews, T Daniel, A I Amin et el. (2012). The clinical significance of radiologically detected indeterminate pulmonary nodules in colorectal cancer. Colorectal Dis. 14, 7, p.828-31.

2. Swensen SJ, Viggiano RW, Midthun DE, et al. (2000). Lung nodule enhancement at CT: multicenter study. Radiology. 214, p.73-80.

3. Gould MK, Maclean CC, Kuschner WG et al. 
(2001). Accuracy of positron emission tomography for diagnosis of pulmonary nodules and mass lesions: a meta-analysis. JAMA. 285, p.914-24.

4. Tang, Kun , Wang et al. (2019). The value of ${ }^{18} \mathrm{~F}-\mathrm{FDG}$ PET/CT in the diagnosis of different size of solitary pulmonary nodules. Medicine. 98, 11 p-e14813 (doi: 10.1097/MD.0000000000014813).

5. Jess $P$, Seiersen $M$, Ovesen $H$ et

(2014). Has PET/CT a role in the characterization of indeterminate lung lesions on staging CT in colorectal cancer? A prospective study. Eur J Surg Oncol. 40, p.719-22.

6. Degirmenci $B$, Wilson $D$, Laymon $C M$ et al. (2008). Standardized uptake value based evaluations of solitary pulmonary nodules using F$18 \quad$ Fluoro-deoxyglucose-PET/computed tomography. Nucl Med Commun. 29, 7, p.614-22.

7. Van Gómez López 0 , García Vicente $A$, Honguero Martínez AF et al. (2015). ${ }^{18}$ F-FDG PET/CT in the assessment of pulmonary solitary nodules: comparison of different analysis methods and risk variables in the prediction of malignancy. Transl Lung Cancer Res. 4, 3, p.228-35.

8. Sang Mi Lee, So Won Oh, Ho-young Lee and Seok-Ki Kim. (2008). FDG PET/CT imaging findings in pulmonary metastases from colorectal cancer. Journal of Nuclear Medicine. 49, 1, p.112.

9. Bamba $Y$, Itabashi M, Kameoka S. (2011). Value of PET/CT imaging for diagnosing pulmonary metastasis of colorectal cancer. Hepatogastroenterology. 58, 112, p.1972-74.

\title{
TÌNH TRANG DINH DƯỡNG VÀ MộT Số YẾU TỐ LIÊN QUAN CỦA TRẺ DƯới 24 THÁNG TUỔI ĐIỀU TRI TẠI KHOA NHI BỆNH VIỆN E
}

\author{
Nguyễn Thị Ngọc Ánh ${ }^{1,2}$, Trương Văn Quý1,2, \\ Nguyễn Thị Diệu Thúy ${ }^{1}$, Nguyễn Quang Dũng ${ }^{1}$
}

\section{TÓM TẮT}

Nghiên cứu được thực hiện trên 121 cặp bà me có con từ 0 đến 24 tháng tuổi điều trị tại khoa Nhi bệnh viện $\mathrm{E}$. Nghiên cứu nhằm đánh giá tình trạng dinh dưỡng và mô tả một số yếu tố liên quan đển tình trạng suy dinh dưỡng (SDD) của trẻ em bằng phương pháp mô tả cắt ngang. Kết quả nghiên cứu cho thây tỷ lệ trẻ SDD thể nhẹ cân $(C N / T)$ là $7,4 \%$; suy dinh dưỡng thể thấp còi $(\mathrm{CC} / \mathrm{T})$ là $9,1 \%$; suy dinh dưỡng thể gâyy còm (CN/CC) là 9,9\%. Trẻ có me trên 35 tuổ có nguy cơ SDD thê gầy còm cao gấp 4,6 lần so với trẻ có mẹ dưới 35 tuổi $(p<0,05)$. Nguy cơ SDD thấp còi ở trẻ sinh ra trong gia đình có từ 2 con trở lên cao hơn nhóm còn lại với $\mathrm{OR}=7,4 ; p<0,05$. Trẻ có cân năng khi sinh $<2500 \mathrm{~g}$ có nguy cơ SDD gây còm và nguy cơ SDD thể nhẹ cân cao hơn trẻ có cân nặng khi sinh từ $2500 \mathrm{~g}$ trở lên với OR lần lượt là 12,3 và 10,4 với $p<0,05$. Chưa thấy mối liên quan có ý nghĩa thống kê giữa nghề nghiệp, kinh tế, trình độ hoc vấn, giới tính trẻ, tuổi của trẻ, cách thức sinh, kiển thức, và thực hành nuôi dương trẻ của bà mẹ với tình trạng suy dinh dưỡng theo các thể của trẻ.

Tư khóa: Trẻ em, suy dinh dưỡng, 0 - 24 tháng.

\section{SUMMARY \\ NUTRIONAL STATUS AND SOME FACTORS RELATED TO MALNUTRITION OF CHILDREN FROM 0 TO 24 MONTHS IN THE PEDIATRIC DERPARTMENT OF E HOSPITAL}

\footnotetext{
${ }^{1}$ Đại học Y Hà nội.

${ }^{2}$ Bềnh viện $E$

Chịu trách nhiệm chính: Nguyễn Thị Diệu Thúy

Email: nguyendieuthuyhmu@gmail.com

Ngày nhận bài: 9.8.2021

Ngày phản biên khoa hoc: 1.1.2021

Ngày duyệt bài: 12.10.2021
}

To asess the nutitional status and some factors related to malnutrition of children from 0 to 24 months in Pediatric department of E hospital. Method: A cross sectional descriptive study with 121 child - mother pairs. Results: The prevalence of manutrition of children from 0 to 24 months was $7,4 \%$ by WAZ; $9,1 \%$ by HAZ and $9,9 \%$ by WHZ. Children whose mothers were over 35 years old have a risk of underweight malnutrition 4.6 times higher than children whose mothers were under 35 years old $(p<0.05)$. The risk of stunting malnutrition in children born in families with 2 or more children is higher than the other group with $\mathrm{OR}=7.4 ; \mathrm{p}<0.05$. Children with birth weight $<2500 \mathrm{~g}$ had a higher risk of stunting malnutrition and underweight malnutrition than children with a birth weight $\geq 2500 \mathrm{~g}$ with Odd ratios of 12.3 and 10.4 respectively with $p<0.05$. There were no statistically significant relationships between occupation, economy, education level and childrearing practices of mothers with malnutrition.

Keywords: Children, malnutrition, 0-24 months

\section{I. ĐĂT VẤN ĐỀ}

Việt Nam trải qua thời kì chiến tranh kéo dài, kinh tế khó khăn với tỷ lệ trẻ suy dinh dưỡng rất cao vào khoảng $50 \%$ trẻ dưới 5 tuổi vào thập niên 80 , đến năm 2010 tỷ lệ suy dinh dưỡng thấp còi là $29,3 \%$, nhẹ cân $17,5 \%^{1}$. Theo báo cáo của tổ chức Alive \& Thrive (A\&T) về tình trạng dinh dưỡng trên 11 tỉnh thành cho thấy tỷ lệ thấp còi ở trẻ dưới 24 tháng tuổi là 9,5\% trong đó $5 \%$ là trẻ dưới 6 tháng tuổi và $15,3 \%$ trẻ từ 6 đến 23,9 tháng tuổi ${ }^{2}$. Ngày càng có nhiều bằng chứng cho thây suy dinh dưỡng trong giai đoạn phát triển quan trọng của con người - trước và trong quá trình mang thai và 